\title{
Análise quantitativa do palato duro de respiradores orais: revisão de literatura
}

\section{Quantitative analysis of the hard palate of mouth breathers: literature review}

\author{
Luana Cristina Berwig ${ }^{1}$, Ana Maria Toniolo da Silva ${ }^{2}$
}

\begin{abstract}
RESUMO
A respiração oral gera inúmeras adaptações patológicas nas estruturas e funções orofaciais, tais como as alterações na morfologia do palato duro. Esta estrutura participa ativamente das funções orais, merecendo destaque na avaliação miofuncional orofacial, que cada vez mais tem lançado mão de instrumentos quantitativos. Apesar de o palato ser de difícil avaliação clínica, pouco se sabe sobre os recursos que podem ser empregados na avaliação quantitativa desta estrutura. Em virtude disso, realizou-se esta revisão de literatura, que teve o objetivo de abordar os resultados das avaliações quantitativas do palato de respiradores orais, bem como os instrumentos que foram empregados nas avaliações. Para tanto, foram revisados periódicos nacionais e internacionais, indexados nas bases de dados Medline, LILACS, Dentistry \& Oral Sciences Source (EBSCOhost) e HighWire Press, entre os anos de 1983 e 2009. Foi possível concluir, a partir da análise dos resultados dos estudos, que a respiração oral se associou com maior frequência ao aumento da profundidade do palato duro e com menor frequência com a diminuição da largura. Além disso, verificou-se que as avaliações do palato duro foram realizadas com diferentes instrumentos de medição, a partir de medidas do palato duro em modelos de gesso, diretamente na boca e em radiografias cefalométricas. Acredita-se que os métodos de avaliação quantitativa possam contribuir para a precisão do diagnóstico fonoaudiológico.
\end{abstract}

Descritores: Palato duro/fisiologia; Medidas; Respiração bucal; Avaliação; Revisão

\section{INTRODUÇÃO}

A respiração nasal permite a proteção das vias aéreas inferiores e o adequado crescimento e desenvolvimento do complexo craniofacial $^{(1-5)}$. A respiração, quando processada através da via nasal, favorece a postura correta dos lábios e da mandíbula. Isso possibilita que a língua fique contida na cavidade oral e em contato com o palato duro, o que estimula a expansão da maxila ${ }^{(1,6)}$.

A respiração nasal pode ser substituída por um padrão de suplência oral. Esta adaptação patológica é comum na prática clínica e afeta tanto as estruturas estáticas quanto as

Trabalho realizado no Curso de Fonoaudiologia, Universidade Federal de Santa Maria - UFSM - Santa Maria (RS), Brasil, com bolsa concedida pela Coordenação de Aperfeiçoamento de Pessoal de Nível Superior (CAPES).

(1) Programa de Pós-Graduação (Mestrado) em Distúrbios da Comunicação Humana, Departamento de Fonoaudiologia, Universidade Federal de Santa Maria - UFSM - Santa Maria (RS), Brasil.

(2) Programa de Pós-Graduação em Distúrbios da Comunicação Humana, Departamento e Curso de Fonoaudiologia, Universidade Federal de Santa Maria - UFSM - Santa Maria (RS), Brasil.

Endereço para correspondência: Luana Cristina Berwig. R. Araújo Viana 173/101, Nossa Senhora de Fátima, Santa Maria (RS), Brasil, CEP: 97015040. E-mail: luanaberwig@gmail.com

Recebido em: 1/10/2010; Aceito em: 23/12/2010 dinâmicas, sendo as modificações no formato do palato duro frequentemente citadas na literatura ${ }^{(2,3,7-13)}$.

O palato duro é parte integrante do sistema estomatognático e participa ativamente das funções de sucção, deglutição, mastigação e fonoarticulação. A homeostase destas funções pode sofrer interferência das alterações morfológicas do palato duro, que muitas vezes decorrem da respiração oral ${ }^{(14)}$.

Apesar de muitos estudos relacionados ao modo respiratório citarem as diferenciações na forma do palato duro, poucos têm enfatizado esta estrutura, principalmente por meio de medidas quantitativas. Em vista disso, acredita-se que o levantamento bibliográfico de estudos da morfologia do palato duro em respiradores orais, que utilizaram medidas objetivas, possibilita a comparação entre o que é referido com base em avaliações clínicas e o que é demonstrado em estudos que avaliaram o palato duro quantitativamente. Além disso, esta revisão de literatura também instiga o conhecimento quanto aos métodos e instrumentos de medição que podem ser empregados na avaliação do palato duro.

Com base no exposto, o objetivo deste estudo foi realizar uma revisão da literatura sobre a análise quantitativa do palato duro em respiradores orais, abordando os resultados das avaliações quantitativas, bem como os instrumentos que foram empregados em tais avaliações. Para tanto, foi realizado 
um levantamento bibliográfico, com periódicos nacionais e internacionais, nas bases de dados Medline, Lilacs, Dentistry \& Oral Sciences Source (EBSCOhost) e HighWire Press.

Foram selecionados os artigos que apresentam medidas quantitativas do palato duro em sua metodologia e que fossem ao encontro da proposta desta revisão de literatura. Assim, dez artigos originais foram encontrados, datados entre 1983 e 2009, dos quais quatro são nacionais e seis internacionais.

\section{REVISÃO DA LITERATURA}

A função respiratória normal é realizada através da via nasal desde o nascimento e assim deve permanecer por toda a vida. Sua função primordial é o preparo do ar para que haja melhor aproveitamento deste nos pulmões. A respiração nasal é fundamental para o crescimento e o desenvolvimento adequados do complexo craniofacial, para a ação correta da musculatura facial e para as funções estomatognáticas ${ }^{(10)}$.

A respiração ideal, através da via nasal, pode ser substituída por um padrão de suplência oral devido a causas obstrutivas como hipertrofia de tonsila faríngea e/ou palatina e a causas não-obstrutivas ou viciosas, por alterações na tensão e postura dos músculos orofaciais, edema transitório da mucosa nasal, obstrução reparada nas vias aéreas, entre outros fatores ${ }^{(5,15)}$.

Muitas adaptações ocorrem no complexo orofacial devido à instalação da respiração oral, tais como alterações na morfologia orofacial e nas funções do sistema estomatognático ${ }^{(5,16)}$. Entre as alterações morfológicas referidas, têm-se as modificações ocorridas no formato do palato duro, sendo que as seguintes nomenclaturas foram encontradas para caracterizar o palato duro de pacientes respiradores orais: palato duro profundo e atrésico ${ }^{(12)}$, palato duro profundo e estreito ${ }^{(3)}$, palato duro ogival e estreito ${ }^{(2,11)}$, palato duro em ogiva ou ogival ${ }^{(7,8,10,13)}$ e palato duro profundo ${ }^{(9)}$.

Quanto aos estudos que realizaram a análise do palato duro por meio de medidas quantitativas, as referências bibliográficas encontradas estão relatadas em ordem cronológica a seguir.

Os primeiros estudos encontrados são dos anos de 1983 e 1987 e verificaram os efeitos da respiração oral por rinite alérgica sobre as características dentofaciais. Estes foram realizados com crianças em fase de dentição mista e entre os aspectos avaliados estava a profundidade do palato duro obtida em radiografias cefalométricas. Os resultados demonstraram que as crianças com respiração oral apresentaram maior profundidade do palato duro quando comparadas às crianças respiradoras nasais, sem rinite alérgica ${ }^{(17,18)}$.

Em 1989, foi publicado um estudo que investigou se na fase de dentição decídua existe alguma alteração esquelética mensurável, associada à respiração oral, através do estudo de modelos em gesso de 30 crianças classificadas como respiradoras nasais e 30 respiradoras orais. Para tanto, foram mensuradas com paquímetro a profundidade do palato duro ao nível dos segundos molares decíduos e as distâncias intercanino e intermolar. O grupo de respiradores orais apresentou distância intercanino menor e profundidade do palato maior do que as crianças respiradoras nasais ${ }^{(19)}$.

No ano de 1999 foram encontrados dois artigos. Um deles verificou a influência da respiração oral sobre a profundidade do palato duro por meio do estudo de modelos em gesso da arcada dentária superior. A amostra foi composta por 30 crianças com modo respiratório nasal e 30 com modo oral, de ambos os gêneros, na faixa etária entre 9 e 14 anos, todas brasileiras e leucodermas. A profundidade do palato duro foi obtida com auxilio de um paquímetro e classificada a partir do cálculo do índice de altura palatina ${ }^{(20)}$, uma relação centesimal entre altura e largura palatina ao nível dos molares, que classifica o palato em camaestafalino (baixo), ortoestefalino (médio) e hipsiestefalino (alto). Os resultados do estudo demonstraram que a profundidade do palato duro das crianças com modo respiratório nasal e oral não diferiram entre $\mathbf{s i}^{(15)}$.

Já o outro estudo, comparou a morfologia da arcada dentária entre as crianças com e sem obstrução respiratória, sendo a profundidade do palato duro um dos aspectos estudados. Esta medida foi realizada com compasso tridimensional em modelos de gesso de crianças com 4 anos de idade. A média da profundidade do palato duro das crianças com obstrução respiratória foi significativamente maior ${ }^{(21)}$.

Em 2001, encontrou-se uma pesquisa que teve o objetivo de comparar a dimensão transversal e vertical do palato duro entre crianças com e sem rinite alérgica, sendo que as mesmas estavam na faixa etária entre 2 e 12 anos. A amostra foi composta por um grupo de 101 crianças com diagnóstico de rinite alérgica recorrente e histórico de respiração oral e um grupo composto por 91 crianças com ausência de patologia e complicações respiratórias. Foi utilizado compasso tridimensional Korkhaus para obtenção de medidas em boca nos plano vertical (profundidade ao nível dos segundos molares decíduos) e tranverso (distância intercanino e intermolar). Não houve diferença entre os grupos quando comparadas as médias das medidas no plano transverso, porém, quando comparadas as médias no plano vertical, observou-se diferenças entre as crianças com e sem rinite alérgica, sendo a profundidade do palato duro maior no primeiro grupo ${ }^{(22)}$.

Em 2005 foram encontrados dois estudos. O primeiro teve como objetivo avaliar a influência da respiração oral na profundidade do palato duro ao nível dos caninos e dos segundos molares decíduos. A amostra foi composta por 99 crianças, de 6 a 8 anos, das quais 29 foram classificadas como respiradoras orais e 70 como respiradoras nasais. Para a realização das medidas de profundidade do palato duro foi confeccionada uma régua milimetrada em acrílico com formato da arcada dentária superior. Os achados deste estudo demonstraram que as crianças respiradoras orais apresentaram palato duro mais profundo ao nível dos molares, enquanto as respiradoras nasais apresentaram maior profundidade ao nível dos caninos ${ }^{(23)}$.

O segundo estudo foi realizado para determinar a influência do vedamento labial incompetente e dos distúrbios respiratórios na morfologia das maloclusões em crianças de 8 a 9 anos. As crianças com vedamento labial incompetente e respiração oral apresentaram média da profundidade palatina maior do que as crianças sem incompetência labial ${ }^{(24)}$.

Em 2008 mais uma pesquisa teve a finalidade de avaliar a dimensão transversal e vertical do palato duro em crianças com rinite alérgica através de um compasso tridimensional Korkhaus. Foram selecionadas 100 crianças, de ambos os gêneros, na faixa etária entre 3 e 12 anos. O grupo de estudo foi 
formado por 50 crianças com rinite alérgica e o grupo controle por 50 crianças sem doenças respiratórias. Neste estudo, não houve diferença nas distâncias intercanino e intermolar entre os grupos controle e estudo, entretanto as crianças com rinite alérgica apresentaram palato duro mais profundo em relação às crianças sem doenças respiratórias ${ }^{(25)}$.

O estudo mais recente foi realizado em 2009 e teve por objetivo investigar as diferenças entre crianças respiradoras nasais e orais quanto às características oclusais e dimensões do palato duro. Com base no diagnóstico otorrinolaringológico, 30 crianças formaram o grupo de respiradoras orais e 30 o grupo de respiradoras nasais, sendo que as mesmas encontravam-se na faixa etária entre 6 e 10 anos. Foram obtidos modelos de gesso das arcadas dentárias, sendo mensuradas, por meio de um compasso tridimensional Korkhaus, a largura do palato duro ao nível dos caninos e a largura e profundidade do palato duro ao nível dos segundos molares decíduos. Os resultados sugeriram que os respiradores orais apresentaram distância

Quadro 1. Avaliação quantitativa do palato duro e comparação dos resultados entre respiradores orais e nasais

\begin{tabular}{|c|c|c|c|}
\hline Autor & Mensuração do palato duro & Resultado para respirador oral & Resultado para respirador nasal \\
\hline $\begin{array}{l}\text { Bresolin et al., } 1983^{(17)} \\
\text { Estados unidos }\end{array}$ & $\begin{array}{l}\text { Medidas em radiografias } \\
\text { cefalométricas }\end{array}$ & $\begin{aligned} P P(6-8 \text { anos }) & =15,7 \mathrm{~mm}^{*} \\
P P(9-12 \text { anos }) & =17,3 \mathrm{~mm}^{*}\end{aligned}$ & $\begin{array}{l}\text { PP (6-8 anos) anos }=14,5 \mathrm{~mm}^{*} \\
\text { PP (9-12anos) anos }=15,8 \mathrm{~mm}^{*}\end{array}$ \\
\hline $\begin{array}{c}\text { Trasks et al., } 1987^{(18)} \\
\text { Estados Unidos }\end{array}$ & $\begin{array}{l}\text { Medidas em radiografias } \\
\text { cefalométricas }\end{array}$ & $\mathrm{PP}=16,74 \mathrm{~mm}^{*}$ & $\mathrm{PP}=14,96 \mathrm{~mm}^{*}$ \\
\hline $\begin{array}{c}\text { Moreira e Lino, 1989(19) } \\
\text { Brasil }\end{array}$ & $\begin{array}{l}\text { Medidas com paquímetro em } \\
\text { modelos de gesso }\end{array}$ & $\begin{array}{c}\mathrm{DIC}=21,3 \mathrm{~mm}^{*} \\
\mathrm{DIM}=27,9 \mathrm{~mm} \\
\text { PP (nível dos } 2^{\text {os }} \text { molares } \\
\text { decíduos) }=13,0 \mathrm{~mm}^{*}\end{array}$ & $\begin{array}{c}\mathrm{DIC}=23,0 \mathrm{~mm}^{*} \\
\mathrm{DIM}=28,8 \mathrm{~mm} \\
\text { PP (nível dos } 2^{\text {os }} \text { molares } \\
\text { decíduos) }=9,6 \mathrm{~mm}^{*}\end{array}$ \\
\hline $\begin{array}{l}\text { Oliveira e Vieira, 1999(15) } \\
\text { Brasil }\end{array}$ & $\begin{array}{l}\text { Medidas em modelos de gesso } \\
\text { e cálculo do índice de altura } \\
\text { palatina }\end{array}$ & $\begin{array}{c}\text { Palato baixo }=6,7 \% \\
\text { Palato médio }=70,0 \% \\
\text { Palato alto }=23,3 \%\end{array}$ & $\begin{array}{l}\text { Palato baixo }=13,4 \% \\
\text { Palato médio }=76,6 \% \\
\text { Palato alto }=10,0 \%\end{array}$ \\
\hline $\begin{array}{l}\text { Löfstrand-Tideström et al., } \\
\qquad \begin{array}{c}1999^{(21)} \\
\text { Suécia }\end{array}\end{array}$ & $\begin{array}{c}\text { Medida com compasso } \\
\text { tridimensional em modelo de } \\
\text { gesso }\end{array}$ & $\begin{array}{l}\text { PP (limite gengival entre os } 1^{\text {os }} \mathrm{e} \\
2^{\text {os }} \text { molares decíduos) }=13,3 \mathrm{~mm}^{*}\end{array}$ & $\begin{array}{l}\text { PP (limite gengival entre os } 1^{\text {os }} \mathrm{e} \\
2^{\text {os }} \text { molares decíduos) }=12,3 \mathrm{~mm}^{*}\end{array}$ \\
\hline $\begin{array}{l}\text { Freitas et al., 2001(22) } \\
\text { Brasil }\end{array}$ & $\begin{array}{l}\text { Medidas com compasso } \\
\text { tridimensional diretamente na } \\
\text { boca }\end{array}$ & $\begin{array}{c}\text { Dentição decídua: } \\
\text { DIC=23,76mm } \\
\text { DIM=29,79mm } \\
\text { PP (nível dos } 2^{\circ s} \text { molares } \\
\text { decíduos) }=11,53 \mathrm{~mm}^{*} \\
\text { Dentição mista: } \\
\text { DIC }=25,45 \mathrm{~mm} \\
\text { DIM=31,09mm } \\
\text { PP (nível dos } 2^{\circ \text { os }} \text { molares } \\
\text { decíduos) }=11,96 \mathrm{~mm}^{*}\end{array}$ & $\begin{array}{c}\text { Dentição decídua: } \\
\text { DIC=23,71mm } \\
\text { DIM=29,57mm } \\
\text { PP (nível dos } 2^{\circ s} \text { molares } \\
\text { decíduos) }=10,0 \mathrm{~mm}^{*} \\
\text { Dentição mista: } \\
\text { DIC }=25,61 \mathrm{~mm} \\
\text { DIM=30,84mm } \\
\text { PP (nível dos } 2^{\circ \mathrm{s}} \text { molares } \\
\text { decíduos) }=10,23 \mathrm{~mm}^{*}\end{array}$ \\
\hline $\begin{array}{l}\text { Perea et al., } 2005^{(23)} \\
\text { Peru }\end{array}$ & $\begin{array}{l}\text { Medidas com régua milimetrada } \\
\text { diretamente na boca }\end{array}$ & $\begin{array}{c}\text { PP (nível dos caninos) }=6,72 \mathrm{~mm}^{*} \\
\text { PP (nível dos } 2^{\text {os }} \text { molares } \\
\text { decíduos) }=17,38 \mathrm{~mm}^{*}\end{array}$ & $\begin{array}{c}\text { PP (nível dos caninos)=7,83mm* } \\
\text { PP (nível dos } 2^{\text {os }} \text { molares } \\
\text { decíduos) }=16,81 \mathrm{~mm}^{*}\end{array}$ \\
\hline $\begin{array}{c}\text { Drevensek e Papic, } 2005^{(24)} \\
\text { Eslovênia }\end{array}$ & $\begin{array}{c}\text { Medidas com compasso } \\
\text { tridimensional em modelos de } \\
\text { gesso }\end{array}$ & $P P=14,1 \mathrm{~mm}^{*}$ & $P P=13,4 \mathrm{~mm}^{*}$ \\
\hline $\begin{array}{c}\text { Ghasempour et al., } 2008^{(25)} \\
\text { Irã }\end{array}$ & $\begin{array}{l}\text { Medidas com compasso } \\
\text { tridimensional diretamente na } \\
\text { boca }\end{array}$ & $\begin{array}{c}\text { Dentição decídua: } \\
\text { DIC=23,7mm } \\
\text { DIM=29,66mm } \\
\text { PP (nível dos } 2^{\text {os }} \text { molares } \\
\text { decíduos) }=10,83 \mathrm{~mm}^{*} \\
\text { Dentição mista: } \\
\text { DIC }=24,91 \mathrm{~mm} \\
\text { DIM=31,97mm } \\
\text { PP (nível dos } 2^{\text {os }} \text { molares } \\
\text { decíduos) }=12,24 \mathrm{~mm}^{*}\end{array}$ & $\begin{array}{c}\text { Dentição decídua: } \\
\text { DIC=23,31mm } \\
\text { DIM=29,58mm } \\
\text { PP (nível dos } 2^{\text {os }} \text { molares } \\
\text { decíduos) }=10,2 \mathrm{~mm}^{*} \\
\text { Dentição mista: } \\
\text { DIC }=24,88 \mathrm{~mm} \\
\text { DIM=32,21 mm } \\
\text { PP (nível dos } 2^{\text {os }} \text { molares } \\
\text { decíduos) }=11,0 \mathrm{~mm}^{*}\end{array}$ \\
\hline $\begin{array}{l}\text { Feres et al., } 2009^{(26)} \\
\text { Brasil }\end{array}$ & $\begin{array}{c}\text { Medidas com compasso } \\
\text { tridimensional em modelo de } \\
\text { gesso }\end{array}$ & $\begin{array}{c}\mathrm{DIC}=23,66 \mathrm{~mm} \\
\mathrm{DIM}=27,61 \mathrm{~mm}^{*} \\
\text { PP (nível dos } 2^{\circ \mathrm{s}} \text { molares } \\
\text { decíduos) }=10,80 \mathrm{~mm}^{*}\end{array}$ & $\begin{array}{c}\mathrm{DIC}=24,03 \mathrm{~mm} \\
\mathrm{DIM}=28,50 \mathrm{~mm}^{*} \\
\text { PP (nível dos } 2^{\text {os }} \text { molares } \\
\text { decíduos) }=9,83 \mathrm{~mm}^{*}\end{array}$ \\
\hline
\end{tabular}


intermolar menor e profundidade do palato duro maior quando comparados aos respiradores nasais ${ }^{(26)}$.

No Quadro 1, foram descritos os procedimentos de realização das medidas do palato duro e os resultados encontrados pelos estudos selecionados para esta revisão de literatura.

\section{DISCUSSÃO}

Na literatura consultada, verificaram-se poucos trabalhos relacionados à avaliação quantitativa do palato duro, sendo que os estudos encontrados utilizaram diferentes métodos de mensuração do palato duro: radiografias cefalométricas ${ }^{(17,18)}$, com paquímetro em modelos de gesso ${ }^{(15,19)}$, com compasso tridimensional em modelos de gesso $^{(21,24,26)}$, com compasso tridimensional diretamente na boca ${ }^{(22,25)}$ e com régua milimetrada diretamente na boca ${ }^{(23)}$.

Foi observado que os estudos realizaram medidas nos planos vertical e/ou transversal. As medidas do plano vertical estão relacionadas à profundidade do palato duro e nas referências consultadas foram realizadas do sulco palatino até o limite gengival dos caninos ${ }^{(23)}$, segundos molares decíduos ${ }^{(19,22,23,25,26)}$, entre os primeiros e segundos molares decíduos ${ }^{(21)}$ e até o limite gengival dos primeiros molares permanentes ${ }^{(15)}$. Alguns estudos não descreveram o limite gengival do dente que serviu de referência para a medida de profundidade ${ }^{(17,18,24)}$. Por sua vez, as medidas do plano transversal caracterizam a largura do palato duro, sendo que na literatura consultada foram realizadas no limite gengival dos caninos ${ }^{(19,22,25,26)}$ e segundos molares decíduos ${ }^{(19,22,25,26)}$.

Os estudos apresentados nesta revisão foram realizados com crianças nas fases de dentição decídua ${ }^{(19,21,22,25)}$ e dentição $\operatorname{mista}^{(15,17,18,22-26)}$, não sendo encontrados estudos com pacientes em fase de dentição permanente.

Na dentição decídua, a partir dos achados com significância estatística apresentados pelos estudos, foi possível inferir que o palato duro das crianças respiradoras orais apresentou maior profundidade na região posterior ${ }^{(19,21,22,25)}$ e menor largura anterior (região dos caninos) ${ }^{(19)}$.

Por sua vez, os resultados com significância estatística apresentados pelas crianças em fase de dentição mista possibilitaram inferir que o palato duro se caracterizou por ser mais profundo na região posterior ${ }^{(22-26)}$, menos profundo anteriormente (região dos caninos) $)^{(23)}$ e mais estreito posteriormente (ao nível dos segundos molares decíduos) ${ }^{(26)}$.

Um dos estudos selecionados foi de encontro aos demais, uma vez que não houve diferença na profundidade do palato duro entre os respiradores orais e nasais na fase de dentição mista. Entretanto, salienta-se que este foi o único estudo encontrado que determinou a profundidade do palato duro através de uma relação centesimal, o que pode justificar tal divergência ${ }^{(15)}$.

Alguns resultados referentes às medidas transversais do palato foram diferentes entre os estudos analisados, o que pode ter sido consequência dos diferentes métodos empregados para o estabelecimento do grupo de respiradores orais, tais como: diagnóstico otorrinolaringológico de respiração oral ${ }^{(26)}$, diag- nóstico de rinite alérgica e histórico de respiração oral ${ }^{(17,18,22,25)}$ e provas clínicas para detecção da respiração oral ${ }^{(15,19,23)}$.

Apesar da maioria dos estudos terem demonstrado que os respiradores orais apresentam maior profundidade e menor largura do palato duro (em algumas medidas), destaca-se que na prática clínica devem ser mantidas as peculiaridades de cada caso. A diferença encontrada nas mensurações do palato duro entre respiradores nasais e orais podem predispor às adaptações das funções estomatognáticas neste segundo grupo, mas isso não pode ser generalizado.

É necessário salientar ainda que esta revisão enfocou a morfologia do palato duro de respiradores orais. Entretanto, outros fatores, como a predisposição genética e hábitos de sucção, podem interferir na configuração desta estrutura.

A partir da revisão realizada, observou-se que não há consenso quanto às terminologias utilizadas na avaliação perceptiva do palato duro ${ }^{(2,3,7-13)}$. Segundo o Comitê de Motricidade Orofacial da Sociedade Brasileira de Fonoaudiologia, o termo palato atrésico deve ser utilizado para se referir ao estreitamento do palato duro e palato ogival é um palato em forma de ogiva, encontrado mais comumente em síndromes ${ }^{(27)}$. Com isso, salienta-se a importância da padronização do uso destas terminologias na avaliação clínica, para que não ocorram equívocos ao se contrastar os resultados encontrados em diferentes estudos.

Com base na literatura consultada, a partir da constatação das alterações do palato duro de respiradores orais, tanto na profundidade quanto na largura, sugere-se o uso de protocolos de avaliação miofuncional orofacial que avaliem clinicamente o palato duro no plano vertical (profundidade) e transversal (largura) ${ }^{(28)}$ e não somente pelo uso de uma única terminologia. Somado a isso, por ser o palato duro uma estrutura de difícil avaliação clínica ${ }^{(29)}$, acredita-se que a inserção das medidas do palato duro, tanto em pesquisas quanto na prática clínica de motricidade orofacial, possam possibilitar maior precisão no diagnóstico.

\section{COMENTÁRIOS FINAIS}

A partir da análise dos resultados dos estudos, foi possível concluir que a respiração oral se associou com maior frequência ao aumento da profundidade do palato duro e com menor frequência à diminuição da sua largura. Para a obtenção destes resultados, diferentes métodos de medição foram empregados, sendo as medidas realizadas em modelos de gesso, em boca ou em radiografias cefalométricas.

Pela importância do palato duro para o funcionamento do sistema estomatognático e pela dificuldade muitas vezes encontrada na avaliação clínica desta estrutura, acredita-se que é preciso refletir quanto à inserção das medidas quantitativas do palato duro, bem como investigar quais os métodos de medição seriam mais aplicáveis em pesquisas e na prática clínica de motricidade orofacial.

Destaca-se que há necessidade de realização de mais pesquisas, principalmente visando estabelecer parâmetros de normalidade quanto às dimensões do palato duro. 


\begin{abstract}
Mouth breathing generates many pathological adaptations in the orofacial structures and functions, such as alterations in the morphology of the hard palate. This structure actively participates in oral functions, deserving attention in the myofunctional orofacial evaluation, which have been increasingly using quantitative instruments. Even though the palate is difficult to be clinically assessed, little is known about the resources that can be used for quantitative evaluation of this structure. For this reason, we carried out this literature review, which had the aim to address the results of quantitative assessments of the palate of mouth breathers, as well as the instruments employed in these assessments. To this end, we reviewed national and international journals indexed in Medline, LILACS, Dentistry \& Oral Sciences Source (EBSCO) and HighWire Press databases between 1983 and 2009. Based on the results of the studies retrieved, we concluded that mouth breathing was more frequently associated to an increase in palatal depth, and less frequently to a decrease in palatal width. Furthermore, it was verified that palatal evaluations used different measurement instruments, based on palatal measures obtained from plaster casts, directly in the mouth, or in cephalometric radiographs. It is believed that the methods of quantitative assessment reviewed can contribute to a precise speech-language pathology diagnosis.
\end{abstract}

Keywords: Palate, hard/physiology; Measures; Mouth breathing; Evaluation; Review

\title{
REFERÊNCIAS
}

1. Andrade FV, Andrade DV, Araújo AS, Ribeiro AC, Deccax LD, Nemr K. Alterações estruturais de órgãos fonoarticulatórios e más oclusões dentárias em respiradores orais de 6 a 10 anos. Rev CEFAC. 2005;7(3):318-25.

2. Rodrigues HO, Faria SR, Paula FS, Motta AR. Ocorrência de respiração oral e alterações miofuncionais orofaciais em sujeitos em tratamento ortodôntico. Rev CEFAC. 2005;7(3):356-62.

3. Bianchini AP, Guedes ZC, Vieira MM. Estudo da relação entre a respiração oral e o tipo facial. Rev Bras Otorrinolaringol. 2007;73(4):500-5.

4. Almeida FL, Silva AM, Serpa EO. Relação entre má oclusão e hábitos em respiradores orais. Rev CEFAC. 2009;11(1):86-93.

5. Hennig TR, Silva AM, Busanello AR, Almeida FL, Berwig LC, Boton LM. Deglutição de respiradores orais e nasais: avaliação clínica fonoaudiológica e eletromiográfica. Rev CEFAC. 2009;11(4):618-23.

6. Tavares JG, Silva EH. Considerações teóricas sobre a relação entre respiração oral e disfonia. Rev Soc Bras Fonoaudiol. 2008;13(4):405-10.

7. Costa JR, Pereira SR, Mitri G, Motta JC, Pignatari SS, Weckx LL. Relação da oclusão dentária com a postura de cabeça e coluna cervical em crianças respiradoras orais. Rev Paul Pediatr. 2005;23(2):88-93.

8. DiFrancesco RC, Bregola EG, Pereira LS, Lima RS. A obstrução nasal e o diagnóstico ortodôntico. Rev Dent Press Ortodon Ortop Facial. 2006;11(1):107-13.

9. Frasson JM, Magnani MB, Nouer DF, Siqueira VC, Lunardi N. Comparative cephalometric study between nasal and predominantly mouth breathers. Rev Bras Otorrinolaringol. 2006;72(1):72-82.

10. Branco A, Ferrari GF, Weber SA. Alterações orofaciais em doenças alérgicas de vias aéreas. Rev Paul Pediatr. 2007;25(3):266-70.

11. Cattoni DM, Fernandes FD, Di Francesco RC, Latorre MR. Características do sistema estomatognático de crianças respiradoras orais: enfoque antroposcópico. Pró-Fono. 2007;19(4):347-51.

12. Barbosa MC, Knop LA, Lessa MM, Araujo TM. Avaliação da radiografia cefalométrica lateral como meio de diagnóstico da hipertrofia de adenóide. Rev Dent Press Ortodon Ortopedi Facial. 2009;14(4):8391.

13. Gouveia SA, Nahás AC, Cotrim-Ferreira FA. Estudo cefalométrico das alterações dos terços médio e inferior da face em pacientes com diferentes padrões respiratórios e tipos faciais. Rev Dent Press Ortodon Ortopedi Facial. 2009;14(4):92-100.

14. Costa TL, Silva HJ, Cunha DA,. A influência da respiração oral na morfologia do palato duro e suas conseqüências no sistema estomatognático. Cesubra Scientia. 2004;1(4):253-64.
15. Oliveira MO, Vieira MM. Influência da respiração bucal sobre a profundidade do palato. Pro-Fono. 1999;11(1):13-20.

16. Perilo TV, Motta AR, Las Casas EB, Saffar JM, Costa CG. Avaliação objetiva das forças axiais produzidas pela língua de crianças respiradoras orais. Rev Soc Bras Fonoaudiol. 2007;12(3):184-90.

17. Bresolin D, Shapiro PA, Shapiro GG, Chapko MK, Dassel S. Mouth breathing in allergic children: its relationship to dentofacial development. Am J Orthod. 1983;83(4):334-40.

18. Trask GM, Shapiro GG, Shapiro PA. The effects of perennial allergic rhinitis on dental and skeletal development: a comparison of sibling pairs. Am J Orthod Dentofacial Orthop. 1987;92(4):286-93.

19. Moreira M, de Paiva Lino A. Evaluation of palatal depth and width in mouth breathers with primary dentition. Int J Orofacial Myology. 1989;15(1):19-24

20. Sicher H, Dubrul EL. O crânio. São Paulo: Artes Médicas; 1991. Anatomia oral; p. 5-94.

21. Löfstrand-Tideström B, Thilander B, Ahlqvist-Rastad J, Jakobsson O, Hultcrantz E. Breathing obstruction in relation to craniofacial and dental arch morphology in 4-year-old children. Eur J Orthod. 1999;21(4):32332.

22. Freitas FC, Bastos EP, Primo LS, de Freitas VL. Evaluation of the palate dimensions of patients with perennial allergic rhinitis. Int J Paediatr Dent. 2001;11(5):365-71.

23. Nieto Perea P, Acosta Quiñones JM, Meneses López A. Determinácion de la profundidad del paladar em niños con respiración bucal de 6-8 años de edad. Rev Estomatol Hered. 2005;15(1):50-3.

24. Drevensek M, Papić JS. The influence of the respiration disturbances on the growth and development of the orofacial complex. Coll Antropol. 2005;29(1):221-5.

25. Ghasempour M, Mohammadzadeh I, Garakani S. Palatal arch diameters of patients with allergic rhinitis. Iran J Allergy Asthma Immunol. 2008;8(1):63-4.

26. Feres MF, Enoki C, Sobreira CR, Matsumoto MA. Dimensões do palato e características oclusais de crianças respiradoras nasais e bucais. Pesqui Bras Odontopediatria Clín Integr. 2009;9(1):25-9.

27. Sociedade Brasileira de Fonoaudiologia. Documento oficial 04/2007 do Comitê de Motricidade Orofacial. São Paulo: Sociedade Brasileira de Fonoaudiologia; 2007.

28. Genaro KF, Berretin-Felix G, Rehder MI, Marchesan IQ. Avaliação miofuncional orofacial: protocolo MBGR. Rev CEFAC. 2009;11(2):23755.

29. Marchesan IQ. Motricidade oral. São Paulo: Pancast; 1993. 